\title{
Menstrual cycle phase does not influence gender differences in experimental pain sensitivity
}

\author{
Rebecca R. Klatzkin ${ }^{b}$, Beth Mechlin ${ }^{b}$, and Susan S. Girdlera,b, \\ aDepartment of Psychiatry, University of North Carolina at Chapel Hill, CB \#7175, Medical Research \\ Bldg A, Chapel Hill, NC 27599-7175, USA \\ bDepartment of Psychology, University of North Carolina at Chapel Hill, CB \#7175, Medical \\ Research Bldg A, Chapel Hill, NC 27599-7175, USA
}

\section{Abstract}

Influence of menstrual cycle phase on experimental pain sensitivity in women and on gender differences in pain sensitivity was examined in 48 men and 49 women in response to cold pressor, heat, and ischemic pain. Each woman was tested at three points in their menstrual cycle in randomized order, the early follicular, late follicular, and luteal phases, while men were also tested three times, controlling for number of days between test sessions. Cycle phase was confirmed via serum hormone levels. As expected, women were significantly more sensitive to cold pain $(p<.01)$, to heat pain $(p<.0001)$, and to ischemic pain $(p<.01)$ than men. However, pain perception during each task was not influenced by the menstrual cycle in women, nor did the menstrual cycle influence the magnitude of the gender differences in pain sensitivity. These results indicate that although women are more sensitive to a variety of noxious stimuli than men, menstrual cycle phase does not appear to moderate those differences in healthy men and women.

\section{Keywords}

Menstrual cycle; Pain sensitivity; Gender differences

\section{Introduction}

\begin{abstract}
It is well established that men and women differ in their perception and experience of pain. In the general population, women report more pain, experience increased severity, frequency, and duration of pain (Unruh, 1996), and are more likely to develop headaches, rheumatoid arthritis, temporomandibular disorders, fibromyalgia, and irritable bowel syndrome than men (Unruh, 1996; Berkley, 1997; Fillingim, 2000). Potential explanations for gender differences in clinical pain can be derived from investigations exploring gender differences in experimental pain perception. A robust finding in the literature is that women display greater pain sensitivity to noxious stimuli than men (Fillingim and Maixner, 1995; Berkley, 1997; Riley et al., 1998; Fillingim, 2000; Shinal and Fillingim, 2007), although the magnitude varies from large to moderate depending on sample size, nature of the stimulus, and whether pain sensitivity is indexed by threshold or tolerance (Riley et al., 1998; Shinal and Fillingim, 2007).
\end{abstract}

\footnotetext{
(C) 2009 European Federation of Chapters of the International Association for the Study of Pain. Published by Elsevier Ltd. All rights reserved.

*Corresponding author. Address: Department of Psychology, University of North Carolina at Chapel Hill, CB \#7175, Medical Research Bldg A, Chapel Hill, NC 27599-7175, USA. Tel.: +1 (919) 966 2544; fax: +1 (919) 966 0708. E-mail address: susan_girdler@med.unc.edu (S.S. Girdler).
} 
The higher prevalence for clinical pain and greater experimental pain sensitivity in women suggest a role for female sex hormones (Fillingim and Ness, 2000; Aloisi, 2003). Analgesic effects of estrogen and progesterone in animals have been documented (Fillingim and Ness, 2000), but consistency in the pattern and direction of the relationship between hormones and nociception is lacking and underlying mechanisms have yet to be elucidated (Sherman and LeResche, 2006). In animals, cyclic variation of ovarian hormones has been shown to affect sensitivity to pain stimuli as well as morphine nociception (Ryan and Maier, 1988; Frye et al., 1992; Martinez-Gomez et al., 1994; Kayser et al., 1996; Sapsed-Byrne et al., 1996; Mogil et al., 2000; Vincler et al., 2001; Vinogradova et al., 2003; Shekunova and Bespalov, 2004; Terner et al., 2005). However, inconsistencies exist regarding which estrous phases are associated with greater nociception (Fillingim and Ness, 2000; Sherman and LeResche, 2006; Craft, 2007).

Methodological issues may also contribute to inconsistent findings on menstrual cycle and pain sensitivity in humans. Despite the consensus of previous literature reviews (Goolkasian, 1980; Riley et al., 1999; Fillingim and Ness, 2000) that menstrual cycle influences pain perception in women, with greatest sensitivity occurring in the ovulatory or luteal phase, a recent review came to a contradictory conclusion. After reviewing 14 studies investigating menstrual cycle effect on pain sensitivity, Sherman and LeResche (2006) concluded that there was no consistent evidence for a menstrual cycle effect on pain perception to a variety of noxious stimuli, with the possible exception of higher pain thresholds to electric pain in the post-ovulatory phase. This review focused on pervasive methodological issues that have limited conclusions from existing studies such as lack of biological markers confirming cycle phase, use of between-subjects designs, small sample sizes, lack of a standardized definition and nomenclature for each cycle phase, and lack of standardized delivery of stimuli (Sherman and LeResche, 2006). Despite inconsistencies regarding phase effects in women, an unanswered question is whether menstrual cycle influences gender differences in pain perception. Consequently, the present study addressed the abovementioned methodological issues in order to examine the influence of cycle phase on gender differences in pain sensitivity.

\section{Methods}

\subsection{Subjects}

Subjects were recruited through newspaper advertisements seeking male and female nonsmokers for a study on pain perception. Of those who responded, 97 met all inclusion/ exclusion criteria and constitute the subjects in this report. The subjects were composed of 48 men and 49 women, aged 18-47 years. Male and female subjects reflected an ethnically diverse sample (49\% African American, 39\% Caucasian, 5\% Indian, 5\% Asian, and 2\% Hispanic). All subjects were medically healthy, with no more than mildly elevated blood pressure (BP) $(<160 / 90 \mathrm{~mm} \mathrm{Hg})$ as determined during an initial screening session. Additionally, subjects were not taking any prescription medication, including oral contraceptives, and not taking any over-the-counter medication on a regular basis (e.g. nonsteroidal anti-inflammatory agents, antihistamines). All women reported regular menstrual cycles (24-32 days). Excluded from participation were subjects with chronic pain conditions (e.g. temporomandibular joint disorder, fibromyalgia, arthritis) and those exhibiting signs of depression or anxiety. Individuals with Hamilton scale (Williams, 1988) depression ratings greater than seven (indicating mild depression) or anxiety ratings greater than nine (indicating symptoms of anxiety) were excluded. The protocol was approved by the institution's Institutional Review Board, and all subjects provided informed, written consent before participating. Subjects received $\$ 500$ compensation. 


\subsection{Procedures}

In order to minimize any influence that novelty of exposure to the laboratory and pain-testing apparatuses would have on pain perception, during an initial screening session subjects were shown the laboratory and the pain-testing equipment, had the procedures described to them, and then underwent a preliminary heat pain threshold assessment.

For all subjects, laboratory testing began between 12 PM and 2 PM. Each woman was tested at three points in her menstrual cycle, early follicular (EF) (days 2-5), late follicular (LF) (days 7-12), and luteal (LUT) (8-12 days after home urine testing revealed the LH surge that precedes ovulation by $24-36 \mathrm{~h}$ ). Order of phase was randomized across female subjects. When no LH surge was detected (indicating anovulation), women were tested in the subsequent luteal phase contingent on the LH surge. Menstrual cycle phase was subsequently confirmed using serum estradiol and progesterone concentrations. Men were also tested three times, matching each consecutive man and woman for number of days between test sessions.

Another goal of the research project was to examine stress-induced analgesia (SIA) (Mechlin et al., 2005). Thus, for each subject and in each cycle phase, pain testing occurred twice, once following the Trier Social Stress Test (TSST) (Kirschbaum et al., 1993) and once following a time equivalent Rest Control period. The order of Stress versus Rest was fully counterbalanced by gender, and for each subject order was held constant over the three test sessions. Although we have previously reported that little evidence of SIA was present in either gender (Mechlin et al., 2005), in order to minimize any potential influence of the mental stressor battery on pain sensitivity, only the results of the pain testing that followed the Rest Control period (see below) are included in this report. It may be important to note that for those subjects who received the Stress Condition first, (50\% of the men and 50\% of the women) a minimum of $1 \mathrm{~h}$ elapsed between the completion of the stress testing and the pain testing that followed the Rest Condition. Thus, given that little evidence for SIA was seen when pain testing immediately followed the mental stress (Mechlin et al., 2005), with at least 60 min elapsing prior to pain testing, it is exceedingly unlikely that stress responses would carry over to influence pain responses following the Rest Condition.

2.2.1. Rest Control period-Immediately prior to pain-testing, subjects rested quietly alone in a comfortable seated position for $20 \mathrm{~min}$.

2.2.2. Pain-testing procedures-Immediately after the Rest Control period, subjects were exposed to the three different pain tests. One of three task orders (i.e. (A) tourniquet, heat, cold; (B) heat, cold, tourniquet; or (C) cold, tourniquet, heat) was randomly assigned to each subject, ensuring that equal numbers of men and women received each of the three orders. This task order remained the same on each of the three testing sessions, and there was a 5 min recovery period following each pain test. Pain intensity and unpleasantness ratings were obtained for each of the three pain tasks. Subjects were instructed that at the point of tolerance for each pain test, they would be asked to rate the intensity and unpleasantness of their pain using separate visual analog scales (0-100). Thus, immediately before deflating the tourniquet cuff, immediately before removal of the hand from the ice bath, and immediately after the third heat tolerance temperature was delivered, the experimenter held up a visual analog scale for intensity rating, with the 100-cm line anchored by the words "not at all intense" and "the most intense pain imaginable." Next, the experimenter held up a scale for unpleasantness, with the $100-\mathrm{cm}$ line anchored by the words "not at all unpleasant" and "the most unpleasant pain imaginable."

2.2.2.1. The submaximal effort tourniquet procedure: In this procedure, as described previously (Maixner et al., 1990), a tourniquet cuff was positioned on the subject's arm and 
the arm placed to the side. Before inflating the tourniquet cuff to $200 \mathrm{mmHg}$ (Hokanson E20 Rapid Cuff Inflator), the subject's arm was raised for $30 \mathrm{~s}$ to promote venous drainage, and then the cuff was inflated, the experimenter's stopwatch started, and the arm returned to the side. To promote forearm ischemia, subjects engaged in 20 handgrip exercises at $30 \%$ of their maximum force with an intersqueeze interval of $2 \mathrm{~s}$. Subjects were instructed to indicate when the sensations in their arm first became painful (pain threshold) and when they were no longer willing or able to tolerate the pain (pain tolerance). A maximum time limit of $20 \mathrm{~min}$ was enforced, though subjects were not informed of this limit.

2.2.2.2. Hand cold pressor: The apparatus for the cold pressor consisted of a container filled with ice and water that was maintained at $4{ }^{\circ} \mathrm{C}$ as recorded immediately before initiating the test. The use of a water circulator prevented the water from warming near the subject's hand. At the onset of the test, subjects were instructed to submerge their hand to the marked line on their wrist and to remain still. Subjects were instructed to indicate to the experimenter when the sensations in their hand first became painful (pain threshold) and to also indicate when they were no longer willing or able to tolerate the pain by saying "stop" (pain tolerance). A maximum time limit of $5 \mathrm{~min}$ was imposed, though subjects were not informed of this limit.

2.2.2.3. Heat pain testing: Heat pain threshold and tolerance were determined by an ascending method of limits using a 1-cm-diameter contact thermode with the capability for a rise time of $10{ }^{\circ} \mathrm{C} / \mathrm{s}$. The thermode was controlled by a personal computer, and heat probe applied to the left volar forearm. During the pain testing, an adapting temperature of $38^{\circ} \mathrm{C}$ was maintained for $10 \mathrm{~s}$. Then, the temperature increased directly to $41.5^{\circ} \mathrm{C}$ and from that point on increased $0.5^{\circ} \mathrm{C}$ every $5 \mathrm{~s}$ until it reached $53^{\circ} \mathrm{C}$ or until the subject reached his/her tolerance. To determine heat pain onset (threshold), subjects were instructed to press a mouse button (which terminated the stimulus) when the heat percept first became painful. This was repeated three times and averaged to calculate heat pain thresholds. Then, three series to determine average heat pain tolerance were conducted by instructing the subject to press a mouse button when they were no longer willing or able to tolerate the pain.

\subsection{Data reduction and analyses}

We first assessed for gender differences in the demographic variables of age and body mass index (BMI) using a one-way analysis of variance (ANOVA) for each variable. We then examined serum estradiol and serum progesterone levels in women across menstrual cycle phase, using a repeated-measure ANOVA, with menstrual cycle phase as the repeated factor. Contrast analyses were performed where significant main effects arose.

Next, we examined menstrual cycle effects in pain threshold and tolerance separately for each of the three pain tasks, using a 2 (Gender) $\times 2$ (Time Point: Threshold/Tolerance) $\times 3$ (Menstrual Cycle Phase) repeated-measures ANOVA, with time point and menstrual cycle phase as repeated factors. Where significant interactions emerged, subsequent simple effects analyses were conducted in order to explore those effects.

We then determined gender and menstrual cycle phase differences in intensity and unpleasantness ratings for each pain task by using a 2 (Gender) $\times 3$ (Menstrual Cycle Phase) repeated-measures ANOVA, with menstrual cycle phase as the repeated factor. ${ }^{1}$ Based on Cohen's measure of effect size (Cohen, 1992), with a sample size of 49 women we had greater than $90 \%$ power to detect a moderate effect size (Cohen's $d=0.50$ ), defined as the difference between pain tolerance levels between each menstrual cycle phase.

\footnotetext{
${ }^{1}$ Finally, in order to assess any spurious differences in pain sensitivity across the 3 testing sessions in males, we examined "cycle phase" differences in men using a repeated measures ANOVA. As expected, no cycle effect was present for males ( $\mathrm{Fs}=0.29-1.31$, ps $=0.28$ $-0.75)$
} 
Finally, with the aim of examining whether any consistent effects of task order on pain threshold and tolerance were present, a 3 (Order: A-tourniquet, heat, cold; B-heat, cold, tourniquet; or C-cold, tourniquet, heat) $\times 3$ (Menstrual Cycle Phase) repeated-measures ANOVA, with menstrual cycle phase as the repeated factor, was performed separately for each time point (threshold and tolerance) and for each of the pain tasks. No robust effect of task order on any measure of pain threshold or tolerance was found (all ps $>.16$ ), with the only significant effect being a main effect of order for cold pressor tolerance $(F(2,94)=4.3, p<$. $05)$, since greater cold pressor tolerance during task order A compared to task order B existed, a difference that was not affected by menstrual cycle phase.

\section{Results}

There were no gender differences in the demographic factors of age or BMI. Females were on average $28( \pm 0.85)$ years of age compared to $27.3( \pm 0.86)$ years of age in males, while the mean BMI for females was $27( \pm 0.92)$, versus $27.4( \pm 0.93)$ in males. As summarized in Table 1, main effects of menstrual cycle phase were found for both estradiol $(F(2,96)=38.73, p<$. $0001)$ and progesterone $(F(2,96)=143.8, p<.0001)$. As expected, contrast analyses for estradiol levels revealed that EF levels were significantly lower than LF levels $(F(1,48)=37.15$, $p<.0001)$, which were significantly lower than LUT levels $(F(1,48)=6.1, p<.05)$. Also as expected, LUT progesterone levels were significantly greater than both $\operatorname{EF}(F(1,48)=144.29$, $p<.0001)$ and LF levels $(F(1,48)=143.49, p<.0001)$, but EF and LF progesterone levels were not significantly different from each other. These results confirm that women were tested at the appropriate points in their menstrual cycle.

Women were significantly more sensitive to cold pain (lower threshold and tolerance) $(F(1,95)$ $=9.57, p<.01)$, to heat pain $(F(1,92)=24.56, p<.0001)$, and to ischemic pain $(F(1,87)=9.0$, $p<.01$ ) than men (Fig. 1, Fig. 2, Fig 3).

Although the anticipated gender differences were present for all three pain tasks, no significant interactions were present involving gender and menstrual cycle phase. Thus, there was no evidence that the female menstrual cycle influenced pain threshold or tolerance in women, nor was there evidence that it influenced the magnitude of the gender differences ${ }^{2}$.

Pain intensity and unpleasantness ratings for all three pain tasks are reported in Table 2. Females had greater cold pain intensity $(F(1,95)=13.76, p<.001)$ and unpleasantness $(F$ $(1,95)=12.5, p<.001)$ ratings than males. No interactions involving gender or menstrual cycle phase were present for cold pain intensity or unpleasantness, therefore gender differences in cold pain intensity and unpleasantness ratings did not vary by menstrual cycle phase. Finally, no main effects or interactions were revealed for heat or ischemic pain intensity or unpleasantness ratings. Thus, despite women showing lower pain thresholds and tolerance values, the genders did not differ in verbal ratings of pain intensity and unpleasantness for either the heat or ischemic pain tests.

\subsection{Secondary analyses examining racexphase effects on pain sensitivity}

In a post-hoc fashion, in order to explore whether the racial diversity of our sample contributed to the absence of a menstrual cycle effect on pain sensitivity in women, we examined menstrual cycle effects in pain threshold and tolerance separately for each of the three pain tasks using a 2 (Race) $\times 2$ (Time Point) $\times 2$ (Menstrual Cycle Phase) repeated-measures ANOVA with time point and menstrual cycle phase as repeated factors, comparing the two primary racial groups, African Americans $(N=25)$, and Non-Hispanic Whites $(N=19)$. Results revealed no

\footnotetext{
${ }^{2}$ The influence of the menstrual cycle on pain threshold and tolerance was not moderated by race (African American vs. non-Hispanic White; Fs $=0.32-1.69$, ps $=0.19-0.72$ )
} 
significant Race $\times$ Phase effects (all ps $>.54$ ), indicating that our racial diversity of the sample did not contribute to the lack of menstrual cycle influence on pain perception reported.

\subsection{Correlations involving sex hormone levels and pain sensitivity in women}

Consistent with the absence of a menstrual cycle effect on pain sensitivity, in women, there was no evidence of meaningful correlations involving estradiol or progesterone levels and pain threshold or tolerance in any cycle phase for any pain task (all rs $=-.27-.14$; all ps $>.05$ ). The only exception was in the early follicular phase involving heat pain threshold and progesterone $(\mathrm{r}=-.34, \mathrm{p}<.05)$. The lack of a consistent pattern suggests that this effect is likely to be spurious.

\section{Discussion}

The results of our study confirm previous findings that women are more sensitive to a variety of laboratory-based noxious stimuli than men (Fillingim and Maixner, 1995; Berkley, 1997;

Riley et al., 1998; Fillingim, 2000), while also suggesting that menstrual cycle phase does not influence pain sensitivity in women, nor does it have an effect on the magnitude of gender differences in pain perception. Initially, the lack of a menstrual cycle effect on pain sensitivity in women may seem contrary to expectations based on previous animal (Ryan and Maier, 1988; Frye et al., 1992; Martinez- Gomez et al., 1994; Kayser et al., 1996; Sapsed-Byrne et al., 1996; Mogil et al., 2000; Vincler et al., 2001; Vinogradova et al., 2003; Shekunova and Bespalov, 2004; Terner et al., 2005) and human research (Goolkasian, 1980; Riley et al., 1999; Fillingim and Ness, 2000). However, recent reviews assessing the association between the estrous cycle and nociception in non-humans show inconclusive results (Fillingim and Ness, 2000; Craft, 2007). Although studies in non-humans generally support an effect of gonadal hormones on sensitivity to noxious stimuli (Goolkasian, 1980; Riley et al., 1999; Fillingim and Ness, 2000), there is no consistent evidence in the pattern and direction of this relationship (Fillingim and Ness, 2000) or a consensus as to which estrous cycle phase is associated with the greatest nociception (Craft, 2007), if any (Ryan and Maier, 1988).

Additionally, our results are supported by a recent study by Kowalczyk et al. (2006) showing no effect of menstrual cycle phase on cold pressor pain threshold and tolerance in normally menstruating women, and by a methodological review concluding that there is no consistent evidence for an effect of the menstrual cycle on the vast majority of laboratory-based noxious stimuli in humans (Sherman and LeResche, 2006). The review by Sherman and LeResche (2006) suggests that lack of methodological rigor in the existing studies on menstrual cycle and pain sensitivity is the basis for the previous inconsistencies reported. In accordance with the suggestions of recent reviews (Sherman and LeResche, 2006; Greenspan et al., 2007) the use of hormonal confirmation of menstrual cycle phase, a within-subjects design, a larger sample size, and three different standardized pain tasks in the current study strongly suggests that, in fact, the female menstrual cycle does not exert a robust effect on pain sensitivity in healthy women, nor does it influence gender differences in pain perception.

Our findings are in contrast, however, to a study published after the Sherman and LeResche (2006) review. Stening et al. (2007) used a within-subjects design and hormonal confirmation of phase, and did find that the late luteal phase was associated with a shorter time to females' report of moderate pain relative to the later follicular phase during the hand cold pressor.

However, cycle phase did not influence pain tolerance or a visual analog scale rating of pain. While the use of a colder ice bath by Stening et al. (2007) relative to the present study $\left(1.5^{\circ} \mathrm{C}\right.$ versus $4.0^{\circ} \mathrm{C}$ ) may have contributed to their cycle effects, the small sample size ( 16 females and 10 males) and lack of detected gender differences in any measure of pain sensitivity limits the conclusions that can be drawn from the Stening et al. (2007) study. 
It may be important to note that in our study, lack of detecting significant cycle effects is unlikely due to insufficient power, as the difference in pain sensitivity across the cycle was minimal (Fig. 1-Fig 3) and we had greater than $90 \%$ power to detect moderate effect sizes by phase had they existed. This is not to say, however, that the menstrual cycle does not influence sensitivity to other laboratory-based noxious stimuli, since Sherman and LeResche (2006) concluded in their review that women may be more sensitive to electrical pain stimulation in the luteal phase. Furthermore, despite the ability of experimental pain sensitivity to predict clinical pain (Fillingim et al., 1996;Edwards et al., 2001), our results should not be interpreted to suggest that the menstrual cycle has no effect on clinical pain severity. A recent literature review on the influence of gonadal hormones on pain suggested that estrogens may modulate some, but not all types of clinical pain (Craft, 2007), while a separate review in chronic pain concluded that higher rates of migraine headache, TMD, and back pain occur at the end of the luteal phase when gonadal hormone levels rapidly decline (Kuba and Quinones-Jenab, 2005).

To our knowledge, this study is the first to examine gender differences in experimental pain sensitivity as a function of female menstrual cycle phase. Similar results to ours (i.e. lack of cycle effect on gender differences in pain perception) have been shown in rodents, since it was found that estrous cycle does not modulate existing sex differences in sensitivity to morphine antinociception (Mogil et al., 2000). While the reasons for a lack of an effect of menstrual cycle phase on gender differences in pain sensitivity are not known, one possibility relates to a putative threshold effect of gonadal hormones on pain sensitivity. For example, even in the early follicular phase of the menstrual cycle when estrogen and progesterone are at their lowest, women still exhibit significantly greater hormone levels than men (Kudolo, 2003). While speculative, it is possible that once a potential threshold level for gonadal hormones is surpassed, relatively minor fluctuation in gonadal hormone levels as occurs over the normal female menstrual cycle would be insufficient to influence gender differences in pain sensitivity. Studies assessing the influence of ovarian suppression with the add-back of exogenous gonadal hormones in a dose-dependent fashion on gender differences in pain perception might shed light on this hypothesis, as might studies using pseudopregnancy models.

Another possibility is that mechanisms other than gonadal hormones are contributing to gender differences in experimental pain sensitivity and may therefore explain why these differences do not fluctuate with the menstrual cycle. For example, cognitive-affective factors such as pain coping strategies, familial factors such as social learning and genetics, endogenous pain mechanisms such as greater analgesic affect of elevated blood pressure in males, and societal sex role expectancies have been suggested as potential factors contributing to gender differences in experimental pain perception (Fillingim, 2000).

In conclusion, the results of our study confirm that although women are more sensitive than men to a variety of noxious stimuli, the menstrual cycle does not exert an influence on pain sensitivity to cold, heat, or ischemic noxious stimuli in women, nor does it moderate gender differences in sensitivity to these pain stimuli. These results are limited, however, to healthy men and women only and may not generalize to women with menstrually related mood disorders, including premenstrual dysphoric disorder, or to other clinical pain populations.

\section{Acknowledgments}

This research was support by NIH grants R01-DA013705 and RO1-MH051246, NIH fellowship MH079532, UNCCH GCRC grant RR0004, and CTSA Grant UL1RR025747.

\section{References}

Aloisi AM. Gonadal hormones and sex differences in pain reactivity. Clin J Pain 2003;19:168-174. [PubMed: 12792555] 
Berkley KJ. Sex differences in pain. Behav Brain Sci 1997;20:371-380. [PubMed: 10097000]

Cohen J. A power primer. Psychol Bull 1992;112:155-159. [PubMed: 19565683]

Craft RM. Modulation of pain by estrogens. Pain 2007;132:S3-S12. [PubMed: 17951003]

Edwards RR, Doleys DM, Fillingim RB, Lowery D. Ethnic differences in pain tolerance: clinical implications in a chronic pain population. Psychosom Med 2001;63:316-323. [PubMed: 11292281]

Fillingim RB. Sex, gender, and pain: women and men really are different. Curr Rev Pain 2000;4:24-30. [PubMed: 10998712]

Fillingim RB, Maixner W. Gender differences in response to noxious stimuli. Pain Forum 1995;4:209_ 221.

Fillingim RB, Maixner W, Kincaid S, Sigurdsson A, Harris MB. Pain sensitivity in patients with temporomandibular disorders: relationship to clinical and psychosocial factors. Clin J Pain 1996;12:260-269. [PubMed: 8969871]

Fillingim RB, Ness TJ. Sex-related hormonal influences on pain and analgesic responses. Neurosci Biobehav Rev 2000;24:485-501. [PubMed: 10817845]

Frye CA, Bock BC, Kanarek RB. Hormonal milieu affects tailflick latency in female rats and may be attenuated by access to sucrose. Physiol Behav 1992;52:699-706. [PubMed: 1329123]

Goolkasian P. Phase and sex effect in pain perception: a critical review. Psychol Women Quart 1980;9:15-28.

Greenspan JD, Craft RM, LeResche L, Arendt-Nielsen L, Berkley KJ, Fillingim RB, et al. Studying sex and gender differences in pain and analgesia: a consensus report. Pain 2007;132:S26-S45. [PubMed: 17964077]

Kayser V, Berkley KJ, Keita H, Gautron M, Guilbaud G. Estrous and sex variations in vocalization thresholds to hindpaw and tail pressure stimulation in the rat. Brain Res 1996;742:352-354. [PubMed: 9117418]

Kirschbaum C, Pirke KM, Hellhammer DH. The 'Trier Social Stress Test'-a tool for investigating psychobiological stress responses in a laboratory setting. Neuropsychobiology 1993;28(1-2):76-81. [PubMed: 8255414]

Kowalczyk WJ, Evans SM, Bisaga AM, Sullivan MA, Comer SD. Sex differences and hormonal influences on response to cold pressor pain in humans. J Pain 2006;7:151-160. [PubMed: 16516820]

Kuba T, Quinones-Jenab V. The role of female gonadal hormones in behavioral sex differences in persistent and chronic pain: clinical versus preclinical studies. Brain Res Bull 2005;66:179-188. [PubMed: 16023915]

Kudolo, G. Reproductive endocrinology. In: Anderson, SC.; Cockayne, S., editors. Clinical chemistry: concepts and applications. McGraw-Hill Professional; 2003. p. 550

Maixner W, Gracely RH, Zuniga JR, Humphrey CB, Bloodworth GR. Cardiovascular and sensory responses to forearm ischemia and dynamic hand exercise. Am J Physiol 1990;259:R1156-R1163. [PubMed: 2260726]

Martinez-Gomez M, Cruz Y, Salas M, Hudson R, Pacheco P. Assessing pain threshold in the rat: changes with estrus and time of day. Physiol Behav 1994;55:651-657. [PubMed: 8190790]

Mechlin MB, Maixner W, Light KC, Fisher JM, Girdler SS. African Americans show alterations in endogenous pain regulatory mechanisms and reduced pain tolerance to experimental pain procedures. Psychosom Med 2005;67:948-956. [PubMed: 16314600]

Mogil JS, Chesler EJ, Wilson SG, Juraska JM, Sternberg WF. Sex differences in thermal nociception and morphine antinociception in rodents depend on genotype. Neurosci Biobehav Rev 2000;24:375-389. [PubMed: 10781697]

Riley JL III, Robinson ME, Wise EA, Myers CD, Fillingim RB. Sex differences in the perception of noxious experimental stimuli: a meta-analysis. Pain 1998;74:181-187. [PubMed: 9520232]

Riley JL III, Robinson ME, Wise EA, Price DD. A meta-analytic review of pain perception across the menstrual cycle. Pain 1999;81:225-235. [PubMed: 10431710]

Ryan SM, Maier SF. The estrous cycle and estrogen modulate stress-induced analgesia. Behav Neurosci 1988;102:371-380. [PubMed: 2840093] 
Sapsed-Byrne S, Ma D, Ridout D, Holdcroft A. Estrous cycle phase variations in visceromotor and cardiovascular responses to colonic distension in the anesthetized rat. Brain Res 1996;742:10-16. [PubMed: 9117383]

Shekunova EV, Bespalov AY. Estrous cycle stage-dependent expression of acute tolerance to morphine analgesia in rats. Eur J Pharmacol 2004;486:259-264. [PubMed: 14985047]

Sherman JJ, LeResche L. Does experimental pain response vary across the menstrual cycle? A methodological review. Am J Physiol Regul Integr Comp Physiol 2006;291:R245-R256. [PubMed: 16484434]

Shinal RM, Fillingim RB. Overview of orofacial pain: epidemiology and gender differences in orofacial pain. Dent Clin North Am 2007;51:1-18. [PubMed: 17185057]

Stening K, Eriksson O, Wahren L, Berg G, Hammar M, Blomqvist A. Pain sensations to the cold pressor test in normally menstruating women: comparison with men and relation to menstrual phase and serum sex steroid levels. Am J Physiol Regul Integr Comp Physiol 2007;293:R1711-R1716. [PubMed: 17652363]

Terner JM, Lomas LM, Picker MJ. Influence of estrous cycle and gonadal hormone depletion on nociception and opioid antinociception in female rats of four strains. J Pain 2005;6:372-383. [PubMed: 15943959]

Vincler M, Maixner W, Vierck CJ, Light AR. Estrous cycle modulation of nociceptive behaviors elicited by electrical stimulation and formalin. Pharmacol Biochem Behav 2001;69:315-324. [PubMed: 11509187]

Vinogradova EP, Zhukov DA, Batuev AS. The effects of stages of the estrous cycle on pain thresholds in female white rats. Neurosci Behav Physiol 2003;33:269-272. [PubMed: 12762594]

Williams JB. A structured interview guide for the Hamilton Depression Rating Scale. Arch Gen Psychiatry 1988;45:742-747. [PubMed: 3395203]

Unruh AM. Gender variations in clinical pain experience. Pain 1996;65:123-167. [PubMed: 8826503] 


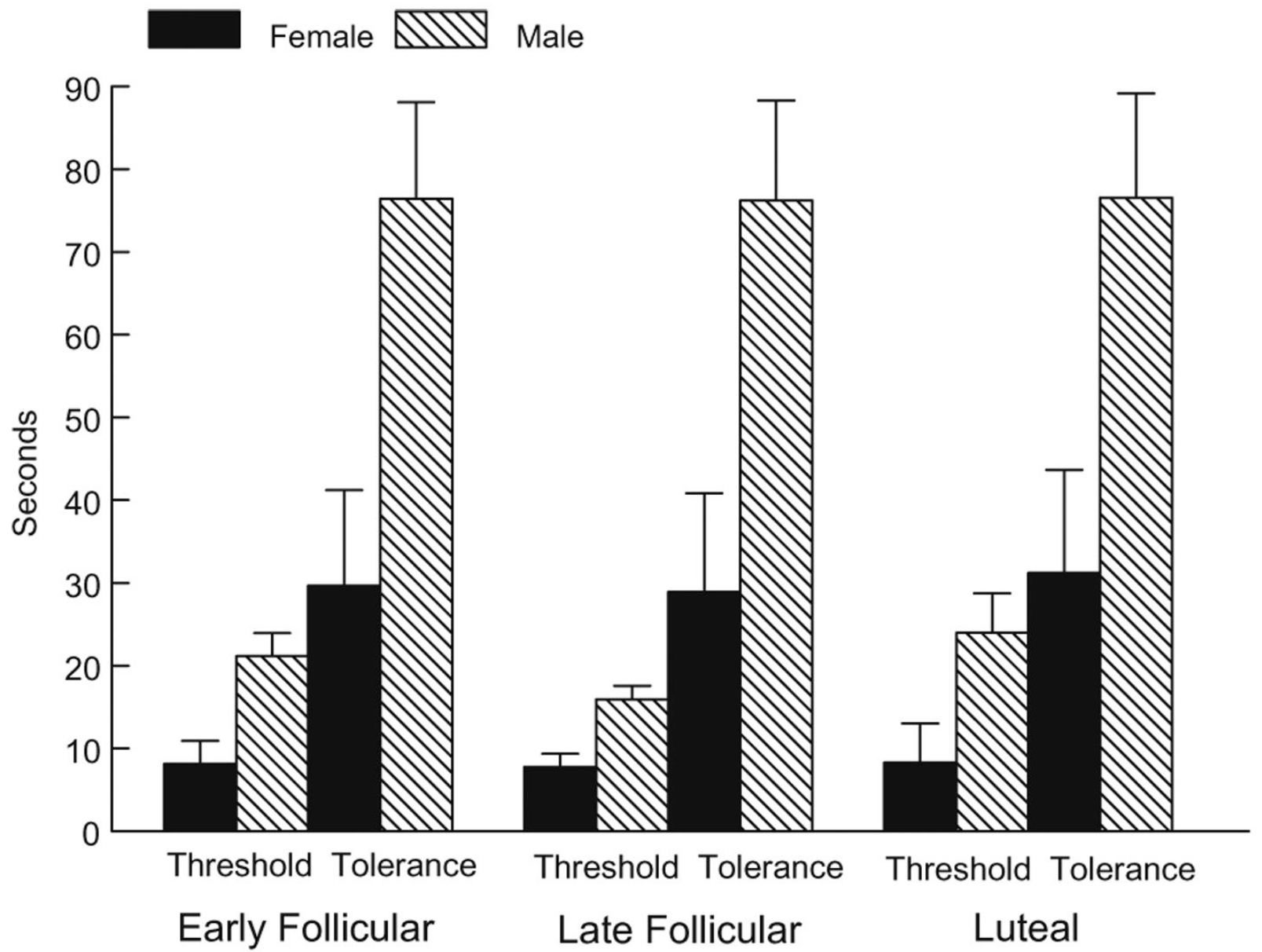

Fig. 1.

Cold pressor pain threshold and tolerance as a function of gender and menstrual cycle phase. 


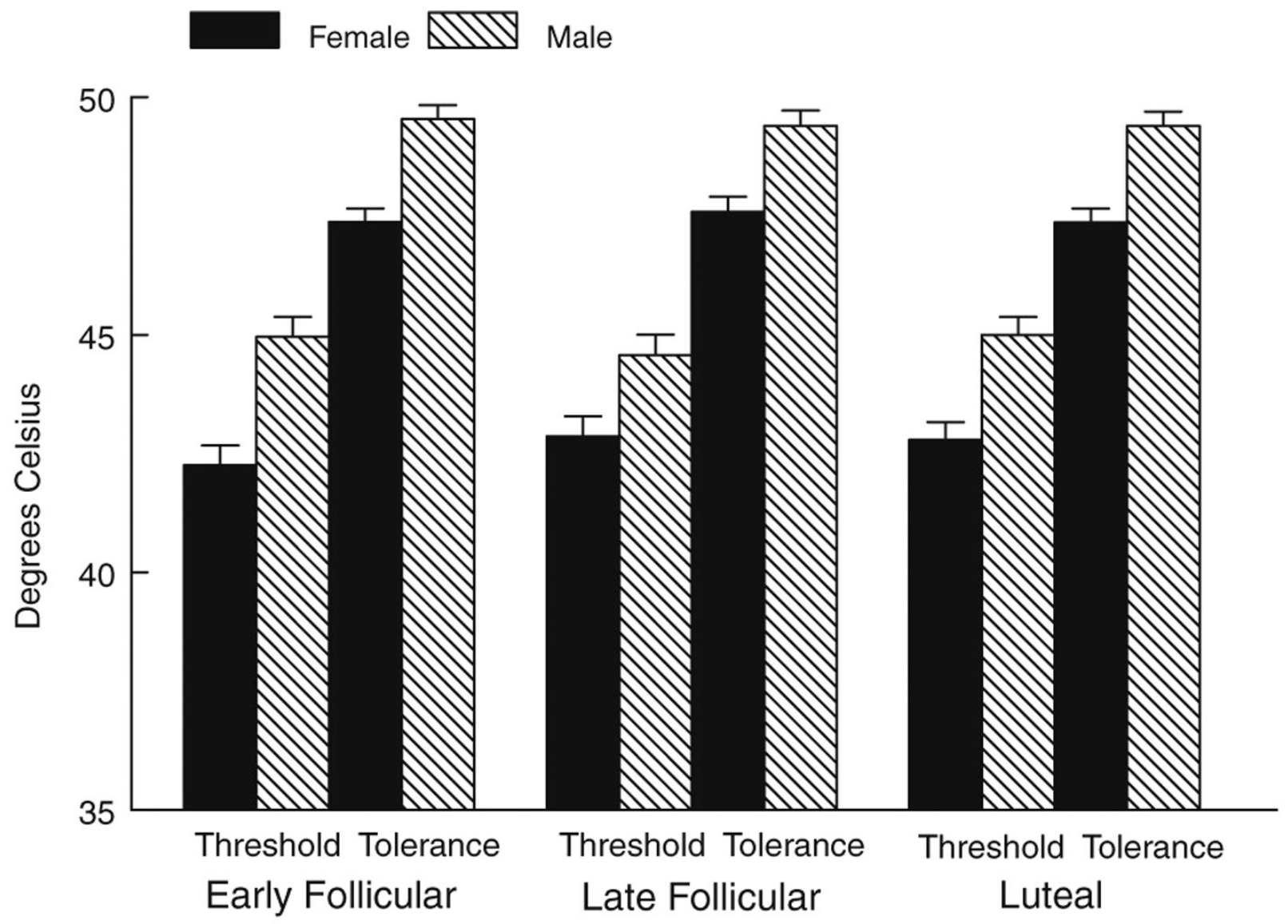

Fig. 2.

Heat pain threshold and tolerance as a function of gender and menstrual cycle phase. 


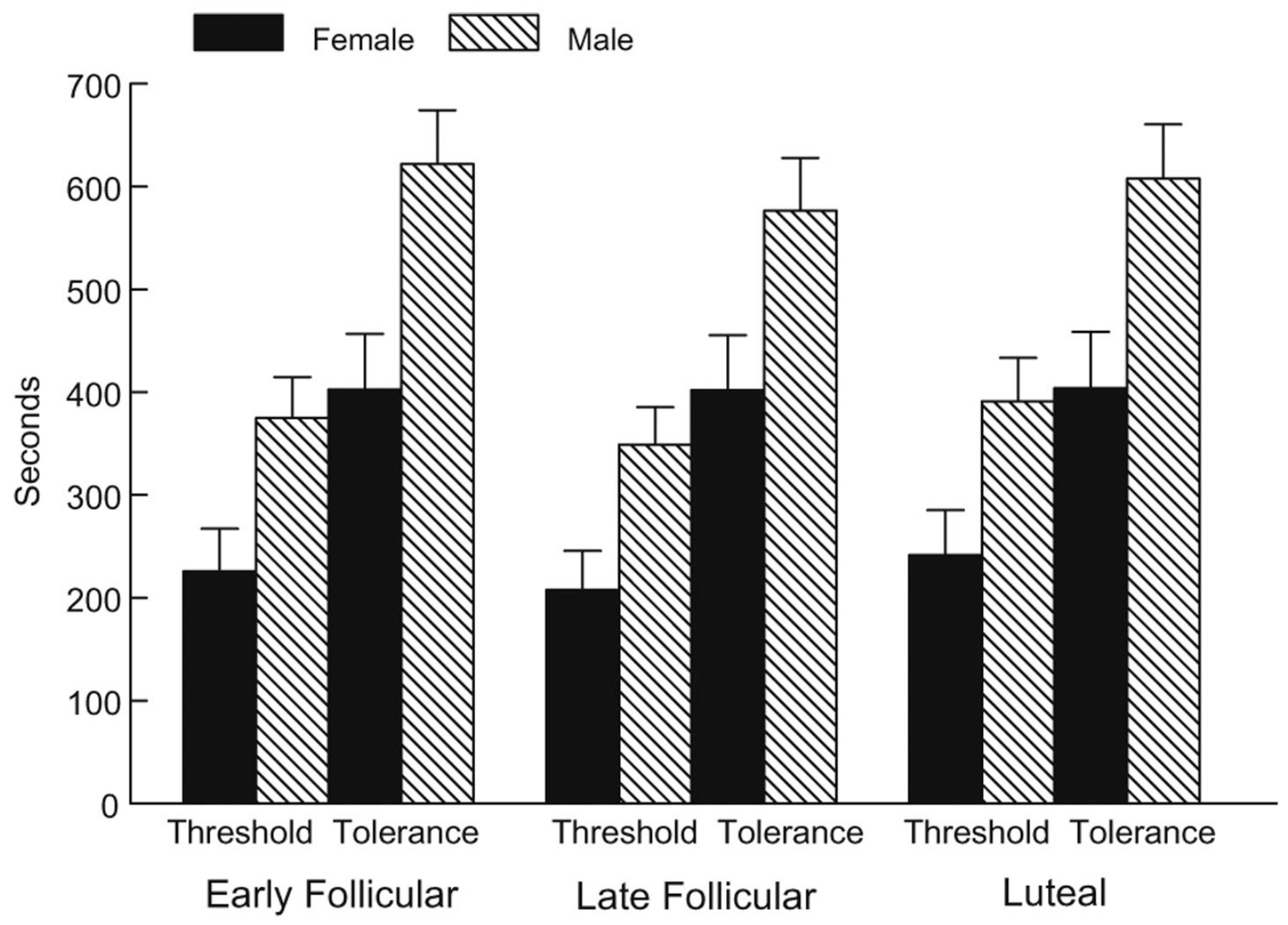

Fig. 3.

Ischemic pain threshold and tolerance as a function of gender and menstrual cycle phase. 


\section{Table 1}

Mean $( \pm$ SEM) estradiol and progesterone values by menstrual cycle phase in women.

\begin{tabular}{lrrl}
\hline & Early follicular $(\mathbf{E F})$ & Late follicular $(\mathbf{L F})$ & Luteal (LUT) \\
\hline Estradiol $(\mathrm{pg} / \mathrm{ml})^{a}$ & $23.25(0.92)$ & $44.27(3.82)$ & $54.75(3.26)$ \\
Progesterone $(\mathrm{pg} / \mathrm{ml})^{b}$ & $0.17(0.02)$ & $0.18(0.04)$ & $13.77(1.1)$ \\
\hline
\end{tabular}

${ }^{a}$ Main effect of menstrual cycle phase, $p<.0001$ : EF $<$ LF $<$ LUT.

${ }^{b}$ Main effect of menstrual cycle phase, $p<.0001: \mathrm{EF}=\mathrm{LF}<\mathrm{LUT}$. 


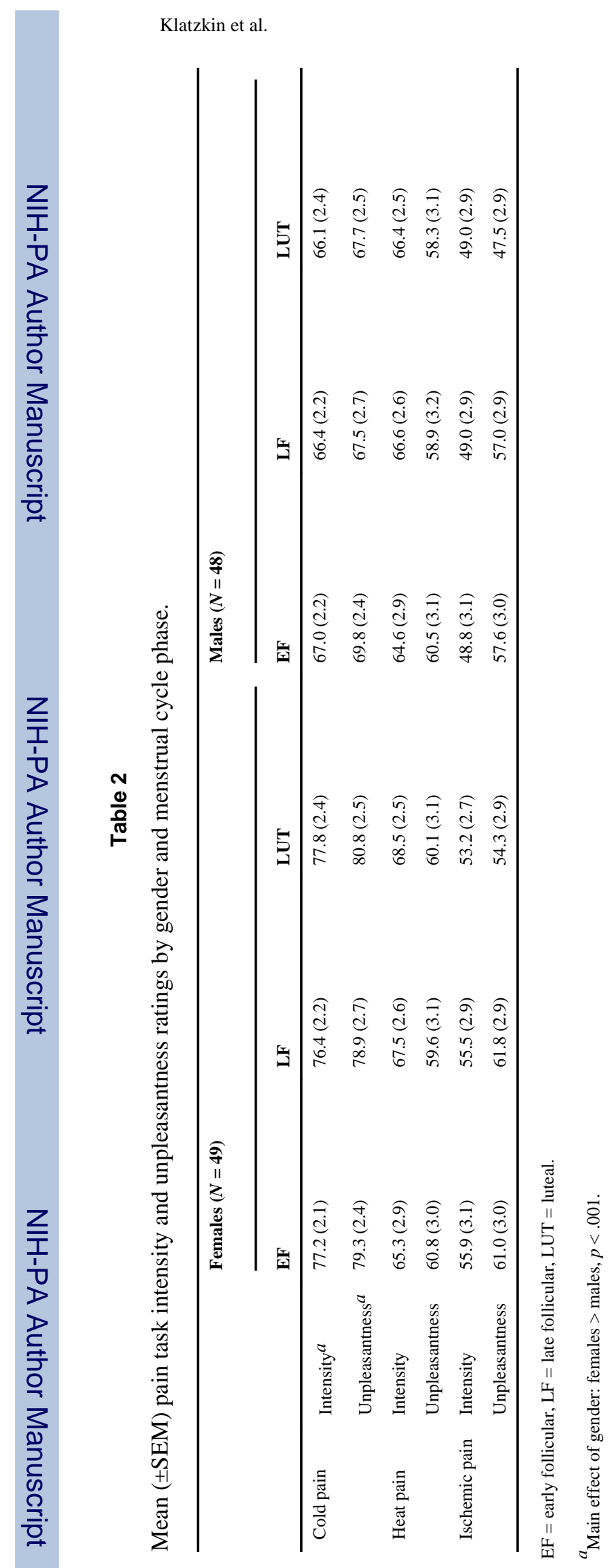

Eur J Pain. Author manuscript; available in PMC 2010 February 10. 